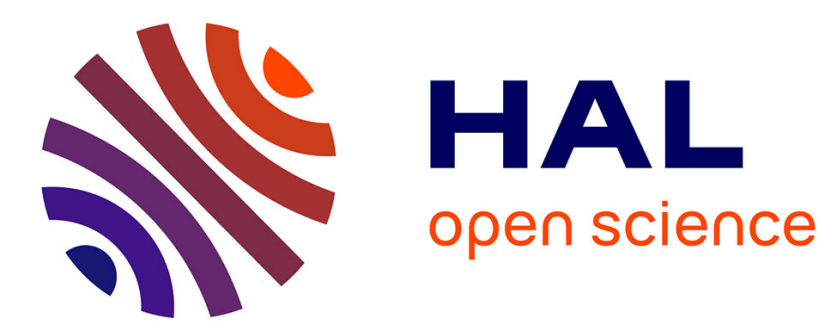

\title{
The absolute trace of totally positive algebraic integers
}

Valérie Flammang

\section{To cite this version:}

Valérie Flammang. The absolute trace of totally positive algebraic integers. International Journal of Number Theory, 2019, 15 (1), pp.173-181. 10.1142/S1793042119500064 . hal-03295884

\section{HAL Id: hal-03295884 \\ https://hal.science/hal-03295884}

Submitted on 22 Jul 2021

HAL is a multi-disciplinary open access archive for the deposit and dissemination of scientific research documents, whether they are published or not. The documents may come from teaching and research institutions in France or abroad, or from public or private research centers.
L'archive ouverte pluridisciplinaire HAL, est destinée au dépôt et à la diffusion de documents scientifiques de niveau recherche, publiés ou non, émanant des établissements d'enseignement et de recherche français ou étrangers, des laboratoires publics ou privés. 


\title{
THE ABSOLUTE TRACE OF TOTALLY POSITIVE ALGEBRAIC INTEGERS
}

\section{FLAMMANG}

\begin{abstract}
Thanks to our recursive algorithm developed in [8], we prove that, if $\alpha$ is a totally positive algebraic integer of degree $d \geq 2$ with minimum conjugate $\alpha_{1}$ then, with a finite number of explicit exceptions,
\end{abstract}

$$
\frac{\operatorname{tr}(\alpha)}{d} \geq \alpha_{1}+1.68
$$

\section{Introduction}

Let $\alpha$ be a totally positive algebraic integer of degree $d \geq 2$ (i.e. its conjugates $\alpha_{1}=\alpha, \ldots, \alpha_{d}$ are all positive real numbers). The trace of $\alpha$ is defined by

$$
\operatorname{tr}(\alpha)=\sum_{i=1}^{d} \alpha_{i}
$$

and we denote by $\mathcal{T}$ the set of all such $\frac{\operatorname{tr}(\alpha)}{d}$.

The Schur-Siegel-Smyth trace problem (so called by P. Borwein in his book [6]) is the following:

Fix $\rho<2$. Then show that all but finitely many totally positive algebraic integers $\alpha$ satisfy $\frac{\operatorname{tr}(\alpha)}{d}>\rho$.

Remark: Solving this problem is equivalent to prove that 2 is the smallest limit point of $\mathcal{T}$.

The problem was solved in 1918 by I. Schur for $\rho<\sqrt{e}[16]$ then in 1945 by C. L. Siegel for $\rho<1.7337$ [17]. The results of Schur and of Siegel involved inequalities on the discriminant of an algebraic integer which is the quantity:

$$
\operatorname{Disc}(\alpha)=\prod_{1 \leq i<j \leq d}\left(\alpha_{i}-\alpha_{j}\right)^{2} .
$$

In 1984, C.J. Smyth solved the problem for $\rho<1.7719$ [18] by using for the first time the principle of auxiliary functions. It lies on the fact that the resultant of two polynomials with integer coefficients and without common factors is a nonzero integer. His idea was at the origin of many works on the subject. In 1997, the author, M. Grandcolas and G. Rhin solved the problem for $\rho<1.7735$ [11]. In 2004, J. McKee and C.J. Smyth for $\rho<1.7783786$ [15], in 2006, J. Aguirre, M. Bilbao and J. C. Peral for $\rho<1.7800$ [1]. In 2007, J. Aguirre and J. C. Peral solved the problem for $\rho<1.7836$ [4] then in 2008 for $\rho<1.784109$ [5]. In all these works, the polynomials involved in the auxiliary functions were found heuristically and have all positive roots. In 2003, Q. Wu [20] linked the auxiliary functions with the integer transfinite diameter ( see Section 3 below) and developed an algorithm which allows a systematic search for relevant polynomials. In 2009, we improved the previous algorithm as described in Section 4 and the polynomials are now found by induction. Hence, we call this new algorithm the recursive algorithm. This led us to solve the problem for $\rho<1.78702$ [8]. J. McKee [14] solved the problem in 2011 for $\rho<1.78839$ using several of our polynomials with complex roots appearing in the auxiliary function. In 2011 also, Y. Liang and Q. Wu [13] solved the problem for $\rho<1.79193$. Finally, in 2016 [7], a slight variant in the use of our recursive algorithm allowed us to improve the known lower bounds and we get : if $\alpha$ is a totally positive algebraic integer of degree $d$ whose 
minimal polynomial is different from $x-1, x^{2}-3 x+1, x^{3}-5 x^{2}+6 x-1, x^{4}-7 x^{3}+13 x^{2}-7 x+1$ and $x^{4}-7 x^{3}+14 x^{2}-8 x+1$ then

$$
\frac{\operatorname{tr}(\alpha)}{d} \geq 1.792812
$$

Besides, J.P. Serre (see Appendix B in [3]) showed that this method does not give such an inequality for any $\rho$ larger than $1.898302 \ldots$ Nevertheless, it is interesting to try to get lower bounds for $\frac{\operatorname{tr}(\alpha)}{d}$. For instance, it was used for the search of Salem numbers of smallest degree with trace equal to -2 by J.F. McKee and C.J. Smyth [15].

Another kind of inequality was established by the author, G. Rhin and C.J. Smyth in 1997 [12]. We got : if $\alpha$ is a totally positive algebraic integer of degree $d \geq 2$ with minimum conjugate $\alpha_{1}$ then, with a finite number of explicit exceptions,

$$
\frac{\operatorname{tr}(\alpha)}{d} \geq 1.6+\alpha_{1}
$$

We used the method of auxiliary functions with heuristic search of "good "polynomials. In 2006, J. Aguirre, M. Bilbao and J C Peral [1] using the same method but adding new polynomials to ours improved the constant 1.6 to the constant 1.66. Now, thanks to our recursive algorithm, we prove the following result:

Theorem 1. Let $\alpha$ be a totally positive algebraic integer of degree $d \geq 2$ with least conjugate $\alpha_{1}$. Then,

$$
\frac{\operatorname{tr}(\alpha)}{d} \geq 1.68+\alpha_{1}
$$

unless $\alpha$ is a zero of one of the polynomials listed in Table 1.

Table 1: List of all monic irreducible polynomials with positive roots, least root $\alpha_{1}$ in $(0,1)$ and Trace/Degree $-\alpha_{1}$ at most 1.68

$\begin{array}{lc}\text { Polynomials P } & \operatorname{tr}(\mathrm{P}) / \operatorname{deg}(\mathrm{P})-\alpha_{1} \\ x^{2}-3 x+1 & 1.1180340 \\ x^{2}-4 x+2 & 1.4142136 \\ x^{2}-5 x+5 & 1.1180340 \\ x^{3}-5 x^{2}+6 x-1 & 1.4686044 \\ x^{3}-6 x^{2}+8 x-2 & 1.6751309 \\ x^{3}-6 x^{2}+9 x-3 & 1.5320889 \\ x^{3}-7 x^{2}+14 x-7 & 1.5803129 \\ x^{3}-8 x^{2}+19 x-13 & 1.4686044 \\ x^{3}-9 x^{2}+24 x-19 & 1.5320889 \\ x^{4}-7 x^{3}+13 x^{2}-7 x+1 & 1.5222229 \\ x^{4}-7 x^{3}+14 x^{2}-8 x+1 & 1.5770909 \\ x^{4}-9 x^{3}+27 x^{2}-31 x+11 & 1.6056743 \\ x^{5}-9 x^{4}+26 x^{3}-29 x^{2}+11 x-1 & 1.6688217 \\ x^{6}-11 x^{5}+42 x^{4}-68 x^{3}+46 x^{2}-12 x+1 & 1.6702846 \\ x^{6}-13 x^{5}+64 x^{4}-151 x^{3}+177 x^{2}-96 x+19 & 1.6703513\end{array}$

This result implies that the only totally positive algebraic integers that satisfy $\frac{\operatorname{tr}(\alpha)}{d}<1.68+\alpha_{1}$ are those whose minimal polynomial belongs to the list above. The proof of this appears in [12] for the constant 1.6.

\section{Proof of Theorem 1}

The proof of the theorem is based on the following result: 
Proposition 1. For every $b \in[0,1)$ there is a polynomial-power $P_{b}=\prod_{j=1}^{J} Q_{j}^{c_{j}}$ where the $Q_{j}^{\prime} s$ are in $\mathbb{Z}[X]$ and the $c_{j}^{\prime}$ s are positive real numbers such that for $x \geq b$,

$$
x-\log \left|P_{b}(x)\right| \geq 1.68+b .
$$

Let $m(b)$ denote the minimum of the function $x-\log \left|P_{b}(x)\right|$ for $x \geq b$. We construct a partition $b_{0}<b_{1}<\ldots<b_{36}=1$ of $[0,1)$ into 35 subintervals such that $m\left(b_{j}\right)-b_{j+1} \geq 1.68(\mathrm{j}=0, \ldots, 35)$. The $b_{j}$ are found recursively. We start with $b_{0}$ equals to 0 and $P_{0}$ is the polynomial-power used in [9] to prove that $\frac{\operatorname{tr}(\alpha)}{d} \geq 1.792812=m(0)$ for all totally positive algebraic integers not a zero of the polynomials making up $P_{0}$. The polynomials involved in $P_{0}$ and their coefficients are available on [10]. Thus, we take $b_{1}=0.1138$. Our recursive algorithm allows us to find a polynomial-power $P_{b_{1}}$ such that $m\left(b_{1}\right)$ is as big as possible. Then the process is iterated. We explain in the following sections how the $P_{b_{j}}$ and the minima $m\left(b_{j}\right)$ are calculated.

The proof of Theorem 1 (originally used in [12]) now follows easily. Let $\alpha$ and $\alpha_{1}$ be as in the statement of the theorem. By replacing $\alpha$ by $\alpha-\left\lfloor\alpha_{1}\right\rfloor$ we can assume that $\alpha_{1} \in(0,1)$. Then we take $b=\alpha_{1}$ in Proposition 1 and we obtain

$$
\frac{\operatorname{tr}(\alpha)}{d} \geq 1.68+\alpha_{1}+\log \left|\prod_{i=1}^{d} P_{\alpha_{1}}\left(\alpha_{i}\right)\right|^{1 / d}
$$

i.e.,

$$
\frac{\operatorname{tr}(\alpha)}{d} \geq 1.68+\alpha_{1}+\left.\sum_{1 \leq j \leq J} c_{j} \log \left|\prod_{i=1}^{d}\right| Q_{j}\left(\alpha_{i}\right)\right|^{1 / d}
$$

where $P_{\alpha_{1}}=\prod_{j=1}^{J} Q_{j}^{c_{j}}$. The polynomials $Q_{j}$ and the exponents $c_{j}$ are available on [10]

Since the minimal polynomial $P$ of $\alpha$ does not divide any $Q_{j}$ then $\prod_{i=1}^{d} Q_{j}\left(\alpha_{i}\right)$ is a nonzero integer because it is the resultant of $P$ and $Q_{j}$.

Hence, if $\alpha$ is not a root of $Q_{j}$, we have

$$
\frac{\operatorname{tr}(\alpha)}{d} \geq 1.68+\alpha_{1}
$$

Table 2: The values of $b$ used in the proof of Proposition 1 and required functions of $m(b)$ 


$\begin{array}{ccc}\text { Run } j & b_{j} & m\left(b_{j}\right) \\ 0 & 0.0000 & 1.792812 \\ 1 & 0.112812 & 1.801901 \\ 2 & 0.121901 & 1.805919 \\ 3 & 0.125919 & 1.809730 \\ 4 & 0.12973 & 1.812613 \\ 5 & 0.132613 & 1.812854 \\ 6 & 0.132854 & 1.812870 \\ 7 & 0.13287 & 1.815242 \\ 8 & 0.135242 & 1.817070 \\ 9 & 0.13707 & 1.821673 \\ 10 & 0.141673 & 1.827923 \\ 11 & 0.147923 & 1.833573 \\ 12 & 0.153573 & 1.842692 \\ 13 & 0.162692 & 1.853991 \\ 14 & 0.173991 & 1.875294 \\ 15 & 0.195294 & 1.900870 \\ 16 & 0.22087 & 1.925140 \\ 17 & 0.24514 & 1.943162 \\ 18 & 0.263162 & 1.957081 \\ 19 & 0.277081 & 1.992052 \\ 20 & 0.312052 & 2.033790 \\ 21 & 0.35379 & 2.088989 \\ 22 & 0.408989 & 2.105574 \\ 23 & 0.425574 & 2.146377 \\ 24 & 0.466377 & 2.162143 \\ 25 & 0.482143 & 2.190407 \\ 26 & 0.510407 & 2.223393 \\ 27 & 0.543393 & 2.246183 \\ 28 & 0.566183 & 2.297433 \\ 29 & 0.617433 & 2.345141 \\ 30 & 0.665141 & 2.367420 \\ 31 & 0.68742 & 2.432224 \\ 32 & 0.752224 & 2.485535 \\ 33 & 0.805535 & 2.552683 \\ 34 & 0.872683 & 2.604163 \\ 35 & 0.924163 & 2.708093 \\ & & \end{array}$

Remark: The following sections reproduce the corresponding sections of [7].

\section{Auxiliary function and generalized integer transfinite diame- ter}

\subsection{Generalized integer transfinite diameter}

Let $K$ be a compact subset of $\mathbb{C}$. The transfinite diameter of $K$ is defined by

$$
\begin{aligned}
& t(K)=\liminf \quad \inf \quad|P|_{\infty, K}^{\frac{1}{n}} \\
& n \geq 1 \quad P \in \mathbb{C}[X] \\
& n \rightarrow \infty \quad P \text { monic } \\
& \operatorname{deg}(P)=n
\end{aligned}
$$

where $|P|_{\infty, K}=\sup _{z \in K}|P(z)|$ for $P \in \mathbb{C}[X]$.

We define the integer transfinite diameter of $K$ by

$$
\begin{array}{ccc}
t_{\mathbb{Z}}(K)= & \liminf _{n \geq 1} \quad \inf _{\substack{n \geq \mathbb{Z}[X] \\
n \rightarrow \infty}} \quad|P|_{\infty, K}^{\frac{1}{n}} \\
& \operatorname{deg}(P)=n
\end{array}
$$

Finally, if $\varphi$ is a positive function defined on $K$, the $\varphi$-generalized integer transfinite diameter 
of $K$ is defined by

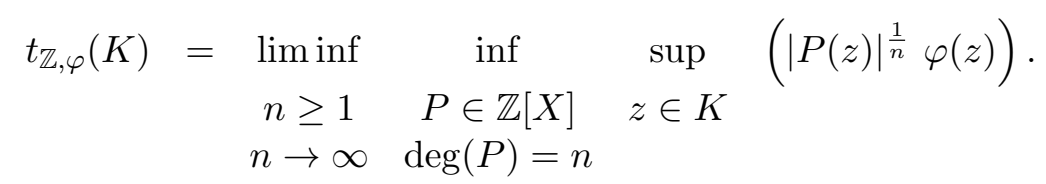

This version of weighted integer transfinite diameter was introduced by F. Amoroso [2] and is an important tool in the study of rational approximations of logarithms of rational numbers.

\subsection{Link with the auxiliary functions}

Let $b$ be in $[0,1)$. The auxiliary function involved here is of the following type:

$$
\text { for } x>b, f(x)=x-\sum_{1 \leq j \leq J} c_{j} \log \left|Q_{j}(x)\right|
$$

where the $c_{j}$ are positive real numbers and the polynomials $Q_{j}$ are nonzero polynomials in $\mathbb{Z}[x]$. In (1), we replace the coefficients $c_{j}$ by rational numbers $a_{j} / q$ where $q$ is a positive integer such that $q . c_{j}$ is an integer for all $1 \leq j \leq J$. Then we can write:

$$
\text { for } x>b, f(x)=x-\frac{t}{r} \log |Q(x)| \geq m
$$

where $Q=\prod_{j=1}^{J} Q_{j}^{a_{j}} \in \mathbb{Z}[X]$ is of degree $r=\sum_{j=1}^{J} a_{j} \operatorname{deg} Q_{j}$ and $t=\sum_{j=1}^{J} c_{j} \operatorname{deg} Q_{j}$ (this formulation was introduced by J. P. Serre). Note that we have $\frac{t}{r}=\frac{1}{q}$. We seek a polynomial $Q \in \mathbb{Z}[X]$ such that

$$
\sup _{x>b}|Q(x)|^{t / r} e^{-x} \leq e^{-m} .
$$

If we suppose that $t$ is fixed, it is equivalent to find an effective upper bound for the weighted integer transfinite diameter over the interval $[b, \infty)$ with the weight $\varphi(x)=e^{-x}$ :

$$
\begin{array}{rccc}
t_{\mathbb{Z}, \varphi}([b, \infty))= & \liminf & \inf & \sup \\
& r \geq 1 & P \in \mathbb{Z}[X] & x>b \\
& r \rightarrow \infty & \operatorname{deg}(P)=r
\end{array}
$$

Remark: Even if we have replaced the compact $K$ by the infinite interval $[b, \infty)$, the weight $\varphi$ ensures that the quantity $t_{\mathbb{Z}, \varphi}([b, \infty))$ is finite.

\section{Construction of an auxiliary function}

The main point is to find a set of "good "polynomials $Q_{j}$, i.e., which gives the best possible value for $m$. Until 2003, the polynomials were found heuristically. For example, in [19] and[4], the authors have searched a collection of polynomials with small absolute trace all of whose roots are positive real numbers. In 2003, Q. Wu [20] has developed an algorithm that allows a systematic search of "good "polynomials. His method was the following. We consider an auxiliary function as defined by (1). We fix a set $E_{0}$ of control points, uniformly distributed on the real interval $I=[b, A]$ where $A$ is "sufficiently large ". Thanks to the LLL algorithm, we find a polynomial $Q$ small on $E_{0}$ within the meaning of the quadratic norm. We test this polynomial in the auxiliary function and we keep only the factors of $Q$ which have a nonzero exponent. The convergence of this new function gives local minima that we add to the set of points $E_{0}$ to get a new set of control points $E_{1}$. We use again the LLL algorithm with the set $E_{1}$ and the process is repeated. 
In 2009 [8], we made two improvements to this previous algorithm in the use of the LLL algorithm. The first one is, at each step, to take into account not only the new control points but also the new polynomials of the best auxiliary function. The second one is the introduction of a corrective coefficient $t$. The idea is to get good polynomials $Q_{j}$ by induction. Thus, we call this algorithm the recursive algorithm. We detail it, always for the trace. The first step consists in the optimization of the auxiliary function $f_{1}=x-t \log x$. We have $t=c_{1}$ where $c_{1}$ is the value that gives the best function $f_{1}$, i.e, whose minimum is as large as possible. We suppose that we have some polynomials $Q_{1}, Q_{2}, \ldots, Q_{J}$ and a function $f$ as good as possible for this set of polynomials in the form (2). We seek a polynomial $R \in \mathbb{Z}[x]$ of degree $k$ (here, $k$ is varying from 15 up to 22 ) such that

$$
\sup _{x \in I}|Q(x) R(x)|^{\frac{t}{r+k}} e^{-x} \leq e^{-m}
$$

where $Q=\prod_{j=1}^{J} Q_{j}$. We want the quantity

$$
\sup _{x \in I}|Q(x) R(x)| \exp \left(\frac{-x(r+k)}{t}\right)
$$

to be as small as possible. We apply the LLL algorithm to the linear forms

$$
Q\left(x_{i}\right) R\left(x_{i}\right) \exp \left(\frac{-x_{i}(r+k)}{t}\right) .
$$

The $x_{i}$ are control points which are points uniformly distributed on the interval $I$ to which we have added points where $f$ has local minima. Thus we find a polynomial $R$ whose irreducible factors $R_{j}$ are good candidates to enlarge the set $\left\{Q_{1}, \ldots, Q_{J}\right\}$. We only keep the factors $R_{j}$ that have a nonzero coefficient in the newly optimized auxiliary function $f$. After optimization, some previous polynomials $Q_{j}$ may have a zero exponent and so are removed.

\section{Optimization of the $c_{j}$}

We have to solve a problem of the following form: find

$$
\max _{C} \min _{x \in X} f(x, C)
$$

where $f(x, C)$ is a linear form with respect to $C=\left(c_{0}, c_{1}, \ldots, c_{k}\right)\left(c_{0}\right.$ is the coefficient of $x$ and is equal to 1 ) and $X$ is a compact domain of $\mathbb{C}$, the maximum is taken over $c_{j} \geq 0$ for $j=0, \ldots, k$. A classical solution consists in taking very many control points $\left(x_{i}\right)_{1 \leq i \leq N}$ and in solving the standard problem of linear programming:

$$
\max _{C} \min _{1 \leq j \leq N} f\left(x_{i}, C\right)
$$

The result depends then on the choice of the control points.

The idea of the semi infinite linear programming (introduced into Number Theory by C. J. Smyth [18]) consists in repeating the previous process adding at each step new control points and verifying that this process converges to $m$, the value of the linear form for an optimum choice of $C$. The algorithm is the following:

(1) We choose an initial value for $C$ i.e., $C^{0}$ and we calculate

$$
m_{0}^{\prime}=\min _{x \in X} f\left(x, C^{0}\right) .
$$

(2) We choose a finite set $X_{0}$ of control points belonging to $\mathrm{X}$ and we have

$$
m_{0}^{\prime} \leq m \leq m_{0}=\min _{x \in X_{0}} f\left(x, C^{0}\right) .
$$


(3) We add to $X_{0}$ the points where $f\left(x, C^{0}\right)$ has local minima to get a new set $X_{1}$ of control points.

(4) We solve the usual linear programming problem:

$$
\max _{C} \min _{x \in X_{1}} f(x, C)
$$

We get a new value for $C$ denoted by $C^{1}$ and a result of the linear programming equal to $m_{1}^{\prime}=\min _{x \in X} f\left(x, C^{1}\right)$. Then we have

$$
m_{0}^{\prime} \leq m_{1}^{\prime} \leq m \leq m_{1}=\min _{x \in X_{1}} f\left(x, C^{1}\right) \leq m_{0}
$$

(5) We repeat the steps from (2) to (4) and thus we get two sequences $\left(m_{i}\right)$ and $\left(m_{i}^{\prime}\right)$ which satisfy

$$
m_{0}^{\prime} \leq m_{1}^{\prime} \leq \ldots \leq m_{i}^{\prime} \leq m \leq m_{i} \leq \ldots \leq m_{1} \leq m_{0},
$$

We stop when there is a good enough convergence, when $m_{i}-m_{i}^{\prime} \leq 10^{-4}$.

Suppose that $p$ iterations are sufficient then we take $m=m_{p}^{\prime}$. 


\section{References}

[1] J. Aguirre, M. Bilbao, J. C. Peral. The trace of totally positive algebraic integers, Math. Comp.,75 (2006), no 253, 385-393.

[2] F. Amoroso. f-transfinite diameter and number theoretic applications, Ann. Inst. Fourier, Grenoble,43, (1993), 1179-1198.

[3] J. Aguirre and J.C. Peral. The Trace Problem for Totally Positive Algebraic Integers, Number Theory and Polynomials. (Conference proceedings, University of Bristol, 3-7 April 2006, editors J.F. McKee and C.J. Smyth). LMS Lecture notes

[4] J. Aguirre and J.C. Peral. The integer Chebyshev constant of Farey intervals, Publ. Mat. 2007, Proceedings of the Primeras Jornadas de Teoría de Números, 11-27.

[5] J. Aguirre and J.C. Peral. The trace problem for totally positive algebraic integers. With an appendix by Jean-Pierre Serre, London Math. Soc. Lecture Note Ser., 352, Number theory and polynomials, 1-19, Cambridge Univ. Press, Cambridge, 2008.

[6] P. Borwein. Computational excursions in analysis and number theory, CMS Books in Mathematics, 10, Springer-Verlag, New York, 2002.

[7] V. Flammang. Une nouvelle minoration pour la trace absolue des entiers algébriques totalement positifs, hal-01346165, 2016.

[8] V. Flammang. Trace of totally positive algebraic integers and integer transfinite diameter, Math. Comp. 78 (2009), no. 266, 1119-1125.

[9] V. Flammang. On the absolute trace of polynomials having all zeros in a sector, Experiment. Math. 17 (2008), no 4, 443-450.

[10] http://www.iecl.univ-lorraine.fr/ Valerie.Flammang/

[11] V. Flammang, M. Grandcolas, G. Rhin. Small Salem numbers, Number Theory in progress, Vol 1 ( Zakopane-Kościelisko, 1997), de Gruyter, Berlin, 1999, 165-168.

[12] V. Flammang, G. Rhin and C. J. Smyth. The integer transfinite diameter of intervals and totally real algebraic integers,J. Théor. Nombres Bordeaux 9 (1997), no. 1, 137-168.

[13] Y. Liang and Q. Wu. The trace problem for totally positive algebraic integers, J. Aust. Math. Soc. 90 (2011), no. 3, 341-354.

[14] J. McKee. Computing totally positive algebraic integers of small trace, Math. Comp. 80 (2011), no 274, 1041-1052.

[15] J. McKee, C.J. Smyth, Salem numbers of trace -2 and traces of totally positive algebraic integers, Algorithmic number theory, Lecture Notes in Computer Science 3076, 327-337, Springer Verlag, Berlin, 2004.

[16] I. Schur. Uber die Verteilung der Wurzeln bei gewissen algebraischen Gleichungen mit ganzzahligen Koeffizienten, Math. Z. 1 (1918), 377-402.

[17] C.L. Siegel. The trace of totally positive and real algebraic integers, Ann. of Maths 46 (1945), 302-312.

[18] C.J. Smyth. Totally positive algebraic integers of small trace, Ann. Inst. Fourrier, Grenoble 33 (1984), 1-28.

[19] C.J. Smyth. Totally positive algebraic integers of small trace, Ann. Inst. Fourrier, Grenoble 33 (1984), 1-28. 
[20] Q. Wu. On the linear independence measure of logarithms of rational numbers, Math. Comp. 72 (2003), 901-911

UMR CNRS 7502. IECL, Université de Lorraine, site de Metz, Département de Mathématiques, UFR MIM, 3 rue Augustin Fresnel, BP 45112, 57073 Metz cedex 03. FRANCE

E-mail address : valerie.flammang@univ-lorraine.fr 